\title{
Determining Cosserat constants of 2D cellular solids from beam models
}

\author{
Stefan Liebenstein ${ }^{1 *}$ (1) and Michael Zaiser ${ }^{1,2}$
}

\author{
*Correspondence: \\ stefan.liebenstein@fau.de \\ ${ }^{1}$ Institute of Materials Simulation \\ (WW8), Friedrich-Alexander \\ University Erlangen-Nürnberg \\ (FAU), Dr.-Mack-Strasse 77, 90762 \\ Fürth, Germany \\ Full list of author information is \\ available at the end of the article
}

\begin{abstract}
We present results of a two-scale model of disordered cellular materials where we describe the microstructure in an idealized manner using a beam network model and then make a transition to a Cosserat-type continuum model describing the same material on the macroscopic scale. In such scale transitions, normally either bottom-up homogenization approaches or top-down reverse modeling strategies are used in order to match the macro-scale Cosserat continuum to the micro-scale beam network. Here we use a different approach that is based on an energetically consistent continuization scheme that uses data from the beam network model in order to determine continuous stress and strain variables in a set of control volumes defined on the scale of the individual microstructure elements (cells) in such a manner that they form a continuous tessellation of the material domain. Stresses and strains are determined independently in all control volumes, and constitutive parameters are obtained from the ensemble of control volume data using a least-square error criterion. We show that this approach yields material parameters that are for regular honeycomb structures in close agreement with analytical results. For strongly disordered cellular structures, the thus parametrized Cosserat continuum produces results that reproduce the behavior of the micro-scale beam models both in view of the observed strain patterns and in view of the macroscopic response, including its size dependence.
\end{abstract}

Keywords: Cellular materials, Disorder, Micro-to-macro transition, Cosserat continuum

\section{Introduction}

Classical constitutive models for elastic materials behavior are local and do not possess intrinsic length scales. For cellular solids the inadequacy of such models is obvious once the relevant scale of a problem becomes comparable to the intrinsic length scale defined by the cell size or strut lengths can be about the same order of magnitude as the system size. As a result smaller samples may behave differently compared to larger ones.

Such size effects can be captured by material models which account for internal length scales, such as constitutive equations in higher order continuum theories (Altenbach et al. 2013). The idea goes back to Cosserat and Cosserat (1909) who enriched the classical formulation by additional independent rotational degrees of freedoms (micro-rotations) and their gradients. Later this was generalized by Eringen (1966); Eringen and Suhubi (1964) to arbitrary, independent micro-deformations. The extended modeling capabilities of such generalized continuum theories come with a cost: the generalized theories require additional boundary conditions and the extended constitutive laws may, in the

(c) The Author(s), 2017 Open Access This article is distributed under the terms of the Creative Commons Attribution 4.0 International License (http://creativecommons.org/licenses/by/4.0/), which permits unrestricted use, distribution, and reproduction in any medium, provided you give appropriate credit to the original author(s) and the source, provide a link to the Creative Commons license, and indicate if changes were made. 
most general cases, involve very significant numbers of material parameters. Experimental determination of these parameters is much more difficult and error-prone compared to the classical theory, efforts have therefore focused on the Cosserat continuum as the simplest case. We mention attempts by Gauthier and Jahsman (1975) for torsion of a circular cylinder as well as a series of studies by Lakes and co-workers (Anderson and Lakes 1994; Chen and Lakes 1991; Lakes 1983; 1986; Yang and Lakes 1982) who investigated cellular materials including bone, polymeric and metallic foams with regards to size effects. More recently Wheel and co-workers (Beveridge et al. 2013; McGregor and Wheel 2014; Wheel et al. 2015) combined experiments and finite element simulations to establish constitutive parameters for Cosserat-type models of heterogeneous materials.

Multi-scale simulation methods which relate a micro-scale model with full resolution of the material microstructure to a generalized continuum macro-model may serve as an alternative or additional approach to experimental parameter identification. For such scale transitions typically two approaches are used. In top-down, reverse modeling approaches, one uses the micro-scale model in very much the same manner as one would deal with experimental data: One measures the (here size-dependent) system-scale response and then tries to fit parameters of the generalized continuum to reproduce the same behavior (see e.g. Diebels and Geringer (2014); Diebels and Steeb (2002); Mora and Waas (2007); Tekoğlu and Onck (2008)). Alternatively, bottom-up homogenization methods can be used to transfer information from the underlying microstructure into the continuum model. Different approaches exist, notably asymptotic methods (Dos Reis and Ganghoffer 2012; Forest et al. 2001; Ghosh et al. 1996), polynomial expansion (Forest 1998; Forest and Sab 1998) and numerical Cauchy-continuum to higher order continuum homogenization (Feyel 2003; Jänicke et al. 2009; Kouznetsova et al. 2004). Many of these approaches are limited to regular or periodic lattices or are computationally expensive and thus not well suited for studies of stochastic systems, where many different microstructures are needed for statistically meaningful averages. The problem is particularly pronounced in analysis of strongly disordered materials where the local response may be controlled by heterogeneous meso-scale stress and strain patterns, which pose inherent problems to homogenization schemes that rely on a certain regularity and macro-homogeneity of the material response.

In this work we focus on two-scale modeling of cellular microstructures of variable disorder where on the microstructure level we represent the materials by networks of Timoshenko beams and on the macroscale by a Cosserat continuum. The basic idea to represent a higher order continuum with lattice models goes back to Woźniak (1970) and was applied in different variations e.g. by Bažant and Christensen (1972); Dos Reis and Ganghoffer (2012); Kumar and McDowell (2004); Nady et al. (2017); Onck (2002); Trovalusci and Pau (2014); Trovalusci et al. (2017). For a review of lattice/beam models in micromechanics see Ostoja-Starzewski $(2002,2007)$. After a brief recap how we create stochastic microstructures we present our own microstructures dependent approach, which we have discussed in more detail elsewhere (Liebenstein et al. 2017). This semianalytical, energy based ansatz is used to obtain, from local stresses and deformations in the discrete model, continuous fields for a micropolar description of the deformation state in terms of displacement gradients, micro- and macro-rotations, and micro-rotation gradients, and of the stress state in terms of the corresponding conjugate stress variables. Linear least square inspired minimization is used for fitting representative material 
parameters to these data. Our investigations cover the spectrum from regular honeycombs to random cellular structures. The resulting constitutive parameters are validated by comparing global spatial stress and strain patterns to those derived from a direct implementation of a Cosserat continuum for compressive and shear loadings of finite samples.

\section{Model}

\section{Computational generation of the microstructure}

Most cellular solids do not possess regular, periodic microstructures such as in honeycombs or other lattice-like structures, but rather exhibit stochastic cellular patterns with varying degree of statistical irregularity. To model the behavior of such stochastic microstructures in a statistically meaningful manner one has to rely on ensembles of statistically equivalent samples, rather than on single realizations. In other words, a comparison of single (or few) realizations across different system sizes is meaningless, as the response of single realizations may be dominated by microstructure fluctuations whereas systematic trends are borne out only upon ensemble averaging. To generate such ensembles we use the approach of Gibson and Ashby (1999); Tekoğlu and Onck (2005); Zhu et al. (2000) to represent the cellular microstructure as a two-dimensional network of Timoshenko beams for which the network structure is generated via Voronoi tessellation of the system plane. The Voronoi tessellation represents reasonably the foaming process under the assumptions (Boots 1982; Van Der Burg et al. 1997) that

1. all nuclei appear simultaneously,

2. the nuclei remain fixed in position throughout the growth process,

3. each nucleus grows isotropically, i.e., at the same rate in all directions,

4. the growth stops for each cell whenever it comes into contact with a neighboring cell.

To tune the degree of irregularity we adopt a method originally proposed by Van Der Burg et al. (1997). As a starting point seeds are generated on a triangular lattice with lattice constant $\Delta p$, which results in a Voronoi tessellation consisting of regular honeycombs. Irregular systems are created by perturbing the position $\boldsymbol{p}$ of each seed by a stochastic vector $\delta \boldsymbol{p}$. In order to obtain a spatially isotropic distribution the direction of the perturbation is chosen isotropically, whereas the perturbation distance $|\delta \boldsymbol{p}|$ is assumed to be exponentially distributed:

$$
f\left(\frac{|\delta \boldsymbol{p}|}{\Delta p}, \beta\right)=\frac{1}{\beta} \exp \left(-\frac{1}{\beta} \frac{|\delta \boldsymbol{p}|}{\Delta p}\right)
$$

The disorder parameter $\beta>0$ defines both the mean value and the standard deviation of the distribution.

Size effects are one main characteristic of materials and models with an intrinsic length scale. In order to faithfully compare different system sizes one must ensure that all systems have the same relative density $\rho_{\text {rel }}$ and the same aspect ratio $R=W / H$ between the system width $W$ and the system height $H$. Under the assumption that all $N_{\mathrm{B}}$ beams have the same in-plane width $w$ and out of plane thickness $t$ but vary in length $l_{i} \gg w$, the total volume covered by the beams is 


$$
V_{\mathrm{B}}=t w \sum_{i=1}^{N_{\mathrm{B}}} l_{i} .
$$

The relative density of a system is

$$
\rho_{\text {rel }}=\frac{V_{\mathrm{B}}}{t W H}=\frac{w}{W H} \sum_{i=1}^{N_{\mathrm{B}}} l_{i},
$$

which is kept constant at $\rho_{\text {rel }}=0.1$ by proportional scaling of $W$ and $H$. As a default aspect ratio for the systems we use the aspect ratio $R_{0}=2 / \sqrt{3}$ of a single regular honeycomb. Each Timoshenko beam is assumed to be linear elastic with Young's modulus $E_{\mathrm{B}}=$ $0.1 \mathrm{GPa}$ and Poisson's ratio $\nu_{\mathrm{B}}=0.3$. The cross-section is quadratic: $t=w=0.05 \Delta p$ with a shear coefficient proposed by Cowper $(1966)$ of $\kappa=10+10 \nu_{\mathrm{B}} /\left(12+11 v_{\mathrm{B}}\right) \approx 0.85$.

Construction of a beam network according to the outlined procedure leads to a single realization of a disordered cellular microstructure with disorder parameter $\beta$. Since we are interested in studying surface effects in finite samples and comparing samples of different disorder, however, a further source of statistical uncertainty needs to be taken into account. The surface response of nearly regular honeycombs strongly depends on the way boundaries are located with respect to the honeycomb lattice. Consider the system shown in Fig. 1 where we have closed, but also barely open honeycombs at the right and left boundaries. Small perturbations of this configuration, i.e. a shift of the structure to the left or right, may open up or close honeycombs at the surfaces. In highly disordered systems, the stiffness changes resulting from such shifts are expected to cancel on average. In regular structures, however, they may significantly alter the overall response. In regular honeycombs such effects may be desirable and even engineered, however, if we want to compare regular with random cellular structures we need to average them out. This can be done by averaging over multiple realizations where we shift the system boundaries by random fractions of the honeycomb lattice period,

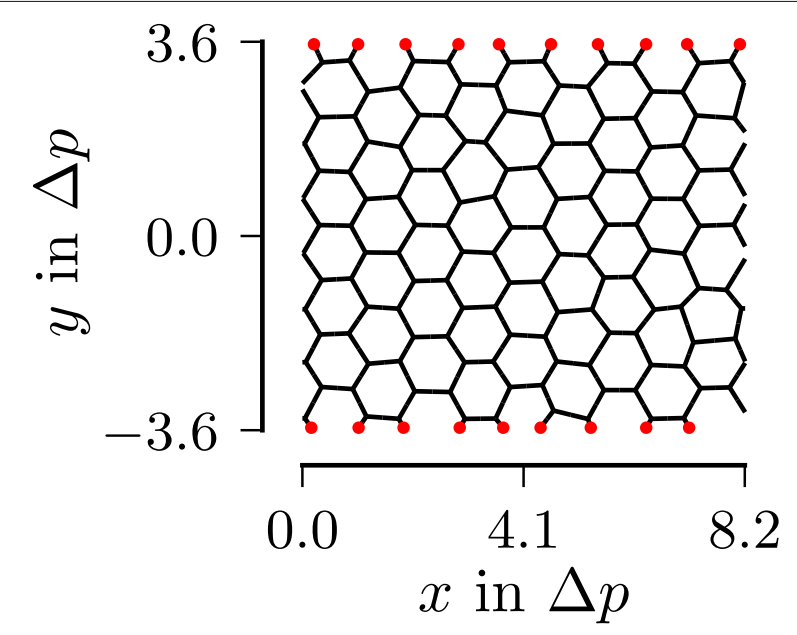

Fig. 1 Microstructure for small irregularity $(\beta=0.05)$, system height $H=7 \Delta p$; boundary conditions are applied at the surface nodes marked in red while the left and right boundaries are free; note that a small shift of the structure to the left would open the cells at the left boundary and considerably reduce the stiffness of the structure under the imposed loading 


$$
\begin{aligned}
& \Delta y=0.5 \Delta p R_{\mathrm{h}}(-1,1), \\
& \Delta x=\left(0.5+\frac{1}{\sqrt{3}}\right) \Delta p R_{\mathrm{w}}(-1,1),
\end{aligned}
$$

where the random numbers $R_{\mathrm{W}}(-1,1), R_{\mathrm{h}}(-1,1)$ are uniformly distributed between -1 and 1 . Such a realization can be envisaged as a randomly located cut-out from a larger system. We therefore speak of random cut-outs when referring to the randomly chosen location of system boundaries with respect to the lattice underlying our beam network construction. A single system realization, hence, consists of a random cut-out from a random microstructure realization.

Each system realization shows a slightly different system response. This poses a problem when comparing with results from the deterministic Cosserat continuum model: On the level of single realizations, we cannot be sure whether differences are systematic (the Cosserat continuum provides an inaccurate or even inadequate representation of the beam systems) or whether they are a mere reflection of the inherent scatter between different beam system realizations. To mitigate this problem, we estimate the scatter of the system response between different realizations and average over a sufficiently large ensemble, with 'sufficiently' defined by the condition that the statistical scatter of the ensemble-averaged response ought to be below $1 \%$ of the mean value of the response. Details are discussed in Appendix A.

In the present work we study two loading cases: displacement driven compression and shear. For a homogeneous continuum, these loadings would correspond to pure uniaxial compression and to simple shear loading. The displacements $\boldsymbol{u}$ and the rotations $\boldsymbol{\phi}$ of the beams at the boundaries (cf. Fig. 1) are given in Table 1 and chosen such that they are comparable to experiments (Andrews et al. 2001; Tekoğlu et al. 2011). The use of displacement controlled boundaries may lead in general (e.g. Chen et al. 1999, and references therein) to a stiffer behavior compared to force controlled boundary conditions. However, Silva et al. (1995) reported, for geometries and boundary conditions similar to those used in the present work, that in large samples where surface effects can be neglected the deviation between force and displacement controlled response becomes small $(<1 \%)$.

It should be noted that from a macroscopic point of view, homogeneous uniaxial compression of an isotropic continuum is not expected to produce higher order/size effects, because uniaxial loading does not induce micro-rotations (Kirchner and Steinmann 2007). On the meso-scale, however, local rotations are always present, and in strongly disordered systems they even might play an important role as a result of structural inhomogeneities. We therefore study both loading cases.

\section{Continuum representaton of stresses and strains in the beam network}

The modeling and finite element simulation of cellular solids as a network of Timoshenko beams gives naturally forces and displacements, evaluated at nodes of the beam elements. To make the transition from this representation at discrete nodal points to a spatially continuous Cosserat continuum we use a method introduced by the authors (Liebenstein

Table 1 Boundary conditions for the two investigated loading conditions

\begin{tabular}{lllll}
\hline Loading & Bottom $(y=-H / 2)$ & Top $(y=H / 2)$ & Left $(x=0)$ & Right $(x=w)$ \\
\hline Compression & $\bar{u}_{y}=-0.025 H$ & $\bar{u}_{y}=0.025 H$ & free & free \\
Simple shear & $\bar{u}_{x}=-0.025 H, \phi=0$ & $\bar{u}_{x}=0.025 H, \phi=0$ & free & free \\
\hline
\end{tabular}


et al. 2017) that is based on the energy equivalence of the beam network and the contiuum. The balance equations for the Cosserat continuum are (see e.g. Eringen (1999); Forest (2009))

$$
\begin{array}{r}
\nabla .(\sigma+S)=\mathbf{0}, \\
\nabla \cdot \mu-S: \epsilon=\mathbf{0},
\end{array}
$$

where $\sigma$ is the symmetric stress and $S$ the skew-symmetric stress tensor which balances the couple stress $\mu$. The symbol $\epsilon$ denotes the Levi-Civita or permutation tensor, $\nabla$ the gradient operator, . is the single contraction and : the double contraction of two tensors. The corresponding stress traction and couple stress traction read

$$
\begin{aligned}
\boldsymbol{t} & =(\boldsymbol{\sigma}+\boldsymbol{S})^{\top} . \boldsymbol{n}, \\
\boldsymbol{m} & =\boldsymbol{\mu}^{\top} . \boldsymbol{n} .
\end{aligned}
$$

With the displacements $\boldsymbol{u}$ and the rotations $\boldsymbol{\omega}$ the corresponding work conjugated strains are related via the strain energy density $W_{c}$

$$
\begin{aligned}
\boldsymbol{\varepsilon} & =\rho \frac{\partial W_{c}}{\partial \boldsymbol{\sigma}}=\frac{1}{2}\left(\nabla \boldsymbol{u}+\nabla \boldsymbol{u}^{\top}\right), \\
\boldsymbol{\varepsilon}^{\mathrm{R}} & =\rho \frac{\partial W_{c}}{\partial \boldsymbol{S}}=\frac{1}{2}\left(\nabla \boldsymbol{u}-\nabla \boldsymbol{u}^{\top}\right)+\boldsymbol{\epsilon} . \boldsymbol{\omega}, \\
\nabla \boldsymbol{\omega} & =\rho \frac{\partial W_{c}}{\partial \boldsymbol{\mu}} .
\end{aligned}
$$

For the derivation of equivalent stresses and strains three major assumptions are made (cf. Liebenstein et al. (2017)): (i) the virtual work $\delta W_{\mathrm{b}}$ of the sum of all beams $N_{\mathrm{b}}$ is the same as the virtual work of the continuum $\delta W_{\mathrm{c}}$ in any given control volume $V_{\mathrm{c}}$, (ii) the displacements and rotations are linear in each control volume and thus stresses and strains are piecewise constant and (iii) the junction point and the centroid of the control volumes are close to each other. Under these assumptions one obtains the following relations for the control volume averages:

$$
\begin{aligned}
\langle\boldsymbol{\sigma}\rangle_{\mathrm{c}} & =\frac{1}{V_{\mathrm{c}}} \int_{V_{\mathrm{c}}} \boldsymbol{\sigma} \mathrm{d} V=\operatorname{sym}\left(\frac{1}{V_{\mathrm{c}}} \sum_{k=1}^{N_{\mathrm{b}}} \boldsymbol{F}^{k} \otimes \boldsymbol{l}^{k}\right), \\
\langle\boldsymbol{S}\rangle_{\mathrm{c}} & =\frac{1}{V_{\mathrm{c}}} \int_{V_{\mathrm{c}}} \boldsymbol{S} \mathrm{d} V=\operatorname{skw}\left(\frac{1}{V_{\mathrm{c}}} \sum_{k=1}^{N_{\mathrm{b}}} \boldsymbol{F}^{k} \otimes \boldsymbol{l}^{k}\right), \\
& =\frac{1}{V_{\mathrm{c}}} \int_{V_{\mathrm{c}}} \boldsymbol{\mu} \mathrm{d} V \\
\langle\boldsymbol{\mu}\rangle_{\mathrm{c}} & =\frac{1}{V_{\mathrm{c}}} \sum_{k=1}^{N_{\mathrm{b}}}\left(\boldsymbol{M}^{k}-\boldsymbol{l}^{k} \times \boldsymbol{F}^{\mathrm{k}}\right) \otimes \boldsymbol{l}^{k},
\end{aligned}
$$

where $\times$ denotes the cross-product, $\boldsymbol{F}$ is the beam force, $\boldsymbol{M}$ the moment acting on a triple (or higher) junction point and $\boldsymbol{l}$ is the so-called beam vector, which is the difference between the midpoint between two junctions and a junction point. The averaged displacement and rotation gradients are for an $N_{c}$-sided control volume calculated from the nodal displacements and rotations as 


$$
\begin{aligned}
\langle\boldsymbol{e}\rangle_{\mathrm{c}} & =\frac{1}{V_{\mathrm{c}}} \int_{V_{\mathrm{c}}} \nabla \boldsymbol{u} \mathrm{d} V \\
& =\frac{1}{V_{\mathrm{c}}} \sum_{k=1}^{N_{\mathrm{c}}}\left(\frac{\boldsymbol{u}^{k}+\boldsymbol{u}^{k+1}}{2} \otimes \boldsymbol{n}^{(k, k+1)}\right) \boldsymbol{b}^{(k, k+1)}, \\
\langle\nabla \boldsymbol{\omega}\rangle_{\mathrm{c}} & =\frac{1}{V_{\mathrm{c}}} \int_{V_{\mathrm{c}}} \nabla \boldsymbol{\omega} \mathrm{d} V \\
& =\frac{1}{V_{\mathrm{c}}} \sum_{k=1}^{N_{\mathrm{c}}}\left(\frac{\boldsymbol{\omega}^{k}+\boldsymbol{\omega}^{k+1}}{2} \otimes \boldsymbol{n}^{(k, k+1)}\right) \boldsymbol{b}^{(k, k+1)},
\end{aligned}
$$

where $\boldsymbol{u}^{k}$ is the displacement and $\omega^{k}$ the rotation of corner node number $k$ in a clockwise enumeration, $b^{(k, k+1)}$ is the length of the polygon side which connects nodes $k$ and $k+1$, and $\boldsymbol{n}^{(k, k+1)}$ is the outward pointing normal vector to this side. From the displacement gradients, strain-like quantities derive in analogy to Eqs. (9), (10) as

$$
\begin{aligned}
\langle\boldsymbol{\varepsilon}\rangle_{\mathrm{c}} & =\frac{1}{2}\left(\langle\boldsymbol{e}\rangle_{\mathrm{c}}+\langle\boldsymbol{e}\rangle_{\mathrm{c}}^{\mathrm{T}}\right), \\
\left\langle\boldsymbol{\varepsilon}^{\mathrm{R}}\right\rangle_{\mathrm{c}} & =\frac{1}{2}\left(\langle\boldsymbol{e}\rangle_{\mathrm{c}}-\langle\boldsymbol{e}\rangle_{\mathrm{c}}^{\top}\right)+\boldsymbol{\epsilon} \cdot\langle\boldsymbol{\omega}\rangle_{\mathrm{c}} .
\end{aligned}
$$

The averaged control volume rotation $\langle\omega\rangle_{\mathrm{c}}$ is calculated by a linear interpolation of the beam-midpoint rotations to be consistent with the constant rotation gradient. In addition to the control volume averages, the system average of a quantity (.) can be approximated as

$$
\langle\cdot\rangle_{\mathrm{S}} \approx \frac{1}{V_{\mathrm{S}}} \sum_{k=1}^{N_{\mathrm{v}}} V_{\mathrm{c}}^{k}\langle\cdot\rangle_{\mathrm{c}}^{k},
$$

where $V_{\mathrm{S}}=\sum_{k=1}^{N_{\mathrm{v}}} V_{c}^{k}$ is the system volume and $N_{\mathrm{v}}$ the number of control volumes in the system.

The control volumes are constructed to match the local microstructure. Each control volume is associated to a triple (or higher) junction in the beam network. The control volume corners are the midpoints between the chosen junction point and the connected neighbor junctions. Additional corner points located at the center points of the Voronoi tessellation ensure that the control volumes provide a tessellation of the entire domain. The exact details of the construction process are given by Liebenstein et al. (2017).

\section{Identifying Cosserat parameters}

Because of the randomness of the underlying microstructure each realization is locally inhomogeneous and also anisotropic regarding its local material properties. A macroscopic representative system however exhibits homogeneous and isotropic material behavior. This means that we can not compare a single realization with a higher order continuum but rather we need to average over an ensemble of many realizations: The ensemble average restores the statistical homogeneity and isotropy of material behavior. For the averaging procedure we first notice that by construction the quantities are piecewise constant in the control volumes which are system specific. To perform averages we need to interpolate them to a common grid. In order to retain the local structure of the stress and strain fields of each realization we use a nearest neighbor interpolation to a fine grid such that there are approximately 6-7 interpolation grid points per control volume. 
This ensures that the localized structure of the stress and strain fields of each realization is preserved as shown in Fig. 2.

The resulting interpolations of a quantity (.) use a common grid and can be directly averaged over all $N_{\mathrm{S}}$ microstructure realizations, and all $N_{\mathrm{CO}}$ cut-outs per realization to analyze the average system response

$$
\langle\cdot\rangle_{\mathrm{E}}=\frac{1}{N_{\mathrm{S}} N_{\mathrm{CO}}} \sum_{N_{\mathrm{S}}} \sum_{N_{\mathrm{CO}}}(\cdot)
$$

As we are using a regular grid the system average of the ensemble average is

$$
\langle\cdot\rangle_{\overline{\mathrm{E}}}=\frac{1}{N_{\mathrm{G}}} \sum_{N_{\mathrm{G}}}\langle\cdot\rangle_{\mathrm{E}},
$$

where $N_{\mathrm{G}}$ is the total number of grid points. Examples of the resulting, ensemble averaged stress field for regular and irregular systems are depicted in Fig. 3.

The averaged system has its counterpart in a higher order continuum for which we want to determine effective material parameters. While the effective classical material parameters can be identified via direct analysis of reaction forces and boundary displacements (similar to Eq. (41)) the Cosserat material constants are not as easily accessible. However, our continuization method computes stress-like $\left\{\langle\boldsymbol{\sigma}\rangle_{\mathrm{c}},\langle\boldsymbol{S}\rangle_{\mathrm{c}},\langle\boldsymbol{\mu}\rangle_{\mathrm{c}}\right\}$ and strain-like quantities $\left\{\langle\boldsymbol{\varepsilon}\rangle_{\mathrm{c}},\left\langle\boldsymbol{\varepsilon}^{\mathrm{R}}\right\rangle_{\mathrm{c}},\langle\nabla \omega\rangle_{\mathrm{c}}\right\}$ independently and thus allows for an identification of material properties. As showed by Gibson and Ashby (1999); Warren and Kraynik (1987), periodic regular honeycombs are (macroscopically) isotropic. Completely stochastic systems of Voronoi cells are expected to be isotropic as well, similar to multi-grain materials, where each grain itself is anisotropic, but on average the system behaves isotropically. Hence we

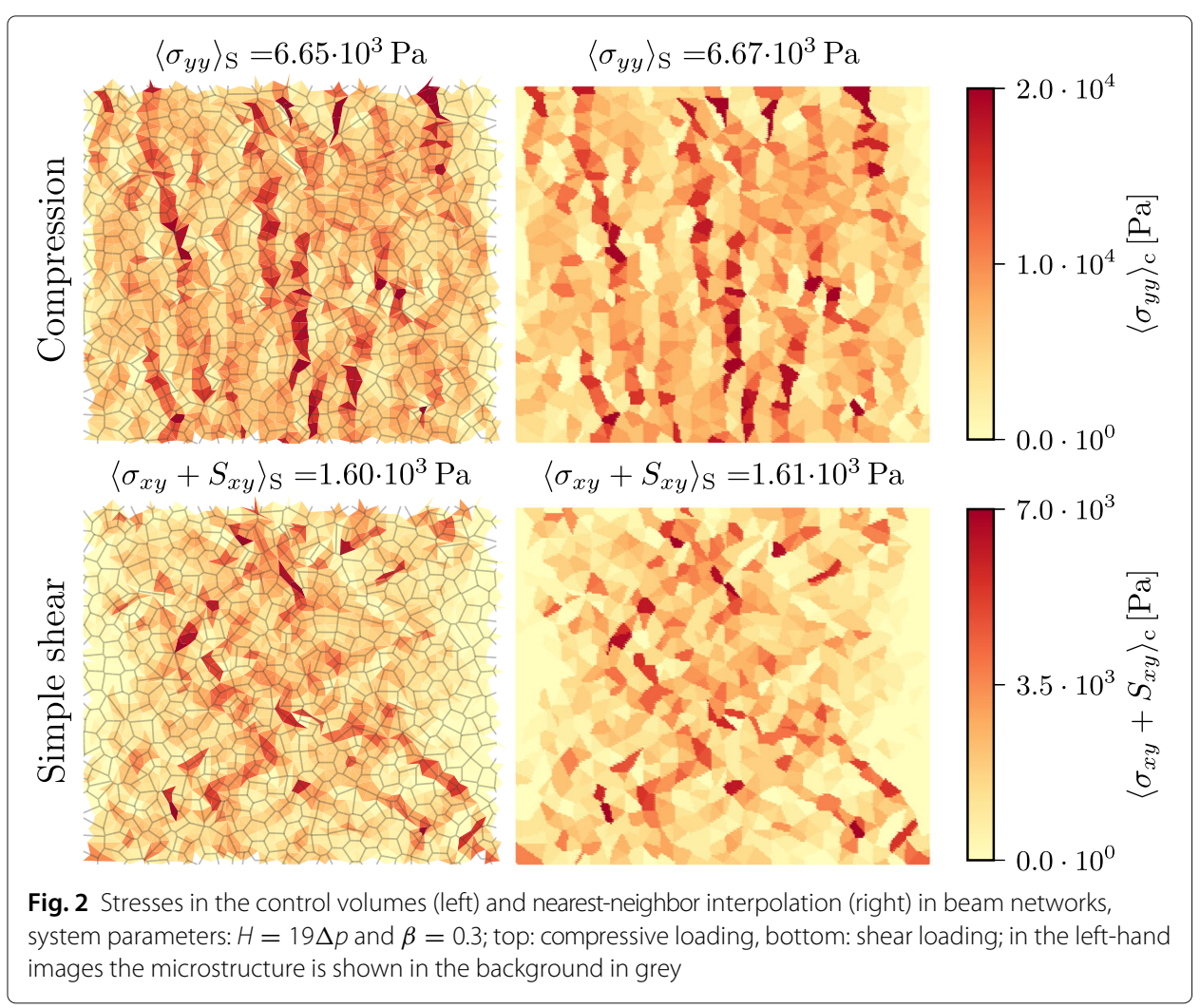




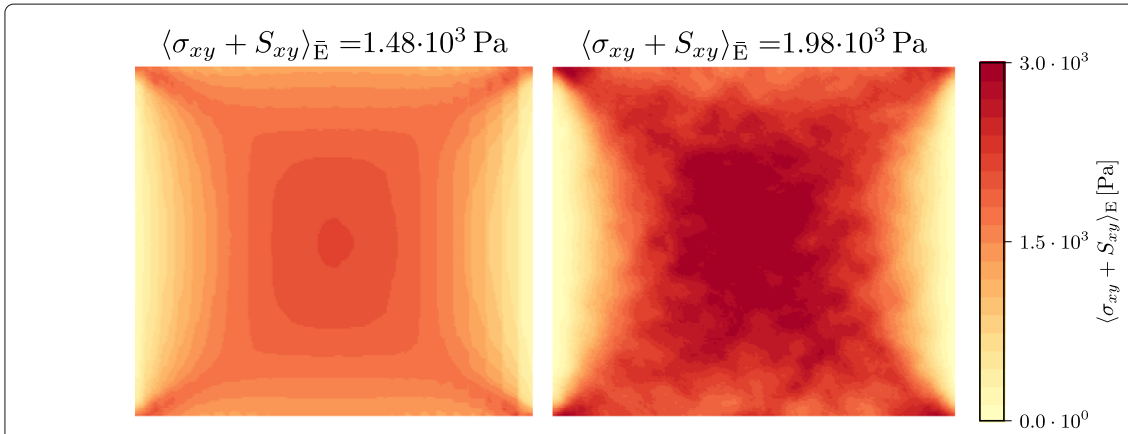

Fig. 3 Averaged system response of beam networks with regular $(\beta=0)$ and irregular $(\beta=5)$ microstructures, simple shear loading by rigid displacement of the top and bottom surface by $\pm 0.025 \mathrm{H}$, system height $H=19$; note the more rigid response of the disordered system

assume, in the limit where length scale effects are unimportant, that the ensemble shows (statistically) isotropic effective material behavior with

$$
\begin{aligned}
& \langle\boldsymbol{\sigma}\rangle_{\overline{\mathrm{E}}}=\mathbb{C}:\langle\boldsymbol{\varepsilon}\rangle_{\overline{\mathrm{E}}}, \\
& \langle\boldsymbol{S}\rangle_{\overline{\mathrm{E}}}=2 G_{\mathrm{C}}\left\langle\boldsymbol{\varepsilon}^{\mathrm{R}}\right\rangle_{\overline{\mathrm{E}}},
\end{aligned}
$$

where $\mathbb{C}$ is the classical isotropic linear elastic stiffness tensor which may be expressed in terms of the Young's modulus $E$ and the Poisson's ratio $v$ in standard manner. $G_{\mathrm{c}}$ is the (isotropic) couple modulus which is a scalar quantity because for the studied 2D structures the $z$-component of the beam vector $\left(l_{z}=0\right)$ as well as the $x$ - and $y$-components of the rotation vector $\left(\omega_{x}=\omega_{y}=0\right)$ are zero - hence, there is only a single axis of rotation. From Eq. (14) it follows that the non-zero components of the couple stress $\boldsymbol{\mu}$ have the same indices as the non-zero entries of the rotation gradient $\nabla \omega$ :

$$
\begin{aligned}
\langle\boldsymbol{\mu}\rangle_{\mathrm{c}} & =\left\langle\mu_{z x}\right\rangle_{\mathrm{c}} \boldsymbol{e}_{z} \otimes \boldsymbol{e}_{x}+\left\langle\mu_{z y}\right\rangle_{\mathrm{c}} \boldsymbol{e}_{z} \otimes \boldsymbol{e}_{y}, \\
\langle\nabla \omega\rangle_{\mathrm{c}} & =\left\langle\omega_{z, x}\right\rangle_{\mathrm{c}} \boldsymbol{e}_{z} \otimes \boldsymbol{e}_{x}+\left\langle\omega_{z, y}\right\rangle_{\mathrm{c}} \boldsymbol{e}_{z} \otimes \boldsymbol{e}_{y} .
\end{aligned}
$$

With that a general, anisotropic relation between the couple stress and the rotation gradient is

$$
\left(\begin{array}{l}
\left\langle\mu_{z x}\right\rangle_{\mathrm{c}} \\
\left\langle\mu_{z y}\right\rangle_{\mathrm{c}}
\end{array}\right)=\underbrace{\left(\begin{array}{cc}
\gamma_{1} & 0 \\
0 & \gamma_{2}
\end{array}\right)}_{\Gamma}\left(\begin{array}{l}
\left\langle\omega_{z, x}\right\rangle_{\mathrm{c}} \\
\left\langle\omega_{z, y}\right\rangle_{\mathrm{c}}
\end{array}\right)
$$

where $\boldsymbol{\Gamma}$ is the anisotropic Cosserat stiffness, which depends on the two additional Cosserat coefficients $\gamma_{1}, \gamma_{2}$. Altogether the parameters which need to be identified are $\mathcal{P}=\left\{E, \nu, G_{\mathrm{c}}, \gamma_{1}, \gamma_{2}\right\}$.

As a method for determining the material constants we apply a fitting approach similar to linear least squares. To obtain effective fitted parameters $\hat{\mathcal{P}}$ we consider the ensemble $\mathcal{E}_{c}$ of all $N_{\mathrm{G}} \times N_{\mathrm{CO}} \times N_{\mathrm{S}}$ gridded control volumes. Over this ensemble we seek to minimize a set of cost functions $\Phi$ with respect to $\mathcal{P}$

$$
\hat{\mathcal{P}}=\underset{\mathcal{P}}{\arg \min } \Phi .
$$

Because $\mathbb{C}(E, v), G_{\mathrm{C}}, \boldsymbol{\Gamma}\left(\gamma_{1}, \gamma_{2}\right)$ are independent of each other we can define and minimize cost function for each set of constants separately. As cost functions the differences 
between measured stresses and the stresses from the multiplication of the elastic constants with the strains are taken and the sum of their squares is minimized:

$$
\begin{aligned}
& \Phi_{\mathbb{C}}=\sum_{\mathcal{E}_{\mathrm{c}}}\left(\langle\boldsymbol{\sigma}\rangle_{\mathrm{c}}-\mathbb{C}:\langle\boldsymbol{\varepsilon}\rangle_{\mathrm{c}}\right):\left(\langle\boldsymbol{\sigma}\rangle_{\mathrm{c}}-\mathbb{C}:\langle\boldsymbol{\varepsilon}\rangle_{\mathrm{c}}\right), \\
& \Phi_{G_{\mathrm{c}}}=\sum_{\mathcal{E}_{\mathrm{c}}}\left(\langle\boldsymbol{S}\rangle_{\mathrm{c}}-2 G_{\mathrm{c}}\left\langle\boldsymbol{\varepsilon}^{\mathrm{R}}\right\rangle_{\mathrm{c}}\right):\left(\langle\boldsymbol{S}\rangle_{\mathrm{c}}-2 G_{\mathrm{c}}\left\langle\boldsymbol{\varepsilon}^{\mathrm{R}}\right\rangle_{\mathrm{c}}\right), \\
& \Phi_{\Gamma}=\sum_{\mathcal{E}_{\mathrm{c}}}\left(\langle\boldsymbol{\mu}\rangle_{\mathrm{c}}-\boldsymbol{\Gamma}:\langle\nabla \boldsymbol{\omega}\rangle_{\mathrm{c}}\right):\left(\langle\boldsymbol{\mu}\rangle_{\mathrm{c}}-\boldsymbol{\Gamma}:\langle\nabla \boldsymbol{\omega}\rangle_{\mathrm{c}}\right) .
\end{aligned}
$$

In order to ensure a positive strain energy density for the 2D (plane strain) case, elastic parameters are restricted to

$$
\begin{array}{lllll}
E>0 & -1>v>1 & G_{\mathrm{c}}>0 & \gamma_{1}>0 & \gamma_{2}>0 .
\end{array}
$$

Numerically this is done via an additional penalty term of the form

$$
\Phi^{\text {pen }}=1000 \mathcal{P}^{4},
$$

which we add if the elastic parameters are outside of the admissible range.

There are, of course, alternative methods for determining elastic constants. A straightforward modification of the above approach is to consider, in defining the cost functions, strain differences and compliances instead of stress differences and stiffnesses. It turns out that this does not lead to any appreciable changes in the resulting elastic constants.

A second alternative method for defining cost functions consists in comparing expressions that directly represent elastic energy density contributions, for instance

$$
\begin{aligned}
\Phi_{\mathbb{C}} & =\sum_{\mathcal{E}_{\mathrm{c}}}\left(\langle\boldsymbol{\varepsilon}\rangle_{\mathrm{c}}: \mathbb{C}:\langle\boldsymbol{\varepsilon}\rangle_{\mathrm{c}}-\langle\boldsymbol{\sigma}\rangle_{\mathrm{c}}: \mathbb{C}^{-1}:\langle\boldsymbol{\sigma}\rangle_{\mathrm{c}}\right)^{2}, \\
\Phi_{G_{\mathrm{c}}} & =\sum_{\mathcal{E}_{\mathrm{c}}}\left(\left\langle\boldsymbol{\varepsilon}^{\mathrm{R}}\right\rangle_{\mathrm{c}}: 2 G_{\mathrm{c}}\left\langle\boldsymbol{\varepsilon}^{\mathrm{R}}\right\rangle_{\mathrm{c}}-\langle\boldsymbol{S}\rangle_{\mathrm{c}}: \frac{1}{2 G_{\mathrm{c}}}\langle\boldsymbol{S}\rangle_{\mathrm{c}}\right)^{2}, \\
\Phi_{\Gamma} & =\sum_{\mathcal{E}_{\mathrm{c}}}\left(\langle\nabla \omega\rangle_{\mathrm{c}}: \boldsymbol{\Gamma}:\langle\nabla \boldsymbol{\omega}\rangle_{\mathrm{c}}-\langle\boldsymbol{\mu}\rangle_{\mathrm{c}}: \boldsymbol{\Gamma}^{-1}:\langle\boldsymbol{\mu}\rangle_{\mathrm{c}}\right)^{2} .
\end{aligned}
$$

This approach yielded broadly similar results which, however, converged much less reliably for strongly disordered systems with high fluctuations in local stresses and strains. Instead of finding the parameters for the effective, averaged systems it might be possible to determine parameters (stiffnesses or compliances) locally for each control volume and then average over all obtained local elastic constants. A problem of this method is that one cannot use the global statistical symmetries of the material and can, therefore, not determine local elastic constants separately for the different loading cases. We leave a further exploration of such direct averaging methods as an open question for future studies.

\section{Results}

As a benchmark for our results we use the well established analytical solutions for regular, periodic honeycombs of Gibson and Ashby (1999). For beams which can deform by bending, axial and shear deformation and have the material properties $\rho_{\text {rel }}=0.1, E_{\mathrm{B}}=$ $1 \cdot 10^{8} \mathrm{~Pa}$, and $\nu_{\mathrm{B}}=0.3$ as used in our simulations, the theoretical Young's modulus, Poisson's ratio and shear modulus are 


$$
\begin{aligned}
& E_{\mathrm{T}}^{*} \approx 1.44 \cdot 10^{5} \mathrm{~Pa} \\
& \nu_{\mathrm{T}}^{*} \approx 0.971, \quad \mathrm{G}_{\mathrm{T}}^{*} \approx 3.65 \cdot 10^{4} \mathrm{~Pa} \text {. }
\end{aligned}
$$

The Cosserat coefficients $\gamma_{i}$ can be reinterpreted in terms of internal lengths $\xi_{i}$ (Diebels and Steeb 2002): $\xi_{i}=\sqrt{\gamma_{i} / 2 G_{\mathrm{c}}}$ with $i=1,2$. The results for three different sizes are shown in Fig. 4. For all sizes the results are very similar which indicates that the computed parameters represent geometry independent material properties. For regular systems, the Young's modulus and Poisson's ratio match very well the analytical solution. As also observed by Zhu et al. (2001) and Liebenstein et al. (2017), increasing irregularity increases the elastic moduli of bulk systems, which can be observed for both loading cases. Poisson's ratio decreases with higher randomness, similar to the result reported by Zhu et al. (2006).

For both loading conditions the couple modulus $G_{\mathrm{c}}$ decreases with increasing degree of irregularity, until the irregular systems have a couple modulus of about $G_{\mathrm{c}} \approx 0.08 G_{\mathrm{T}}^{*}$. These results are consistent with experimental data quoted in a recent review by Hassanpour and Heppler (2017) who indicate values in the range of $G_{\mathrm{C}} \approx 0.1 G_{\mathrm{T}}^{*}$ for polystyrene foams, syntactic foams and a polymethacrylimide foam. The internal length $\xi$ shows an anisotropic behavior for regular systems. For both loadings considered, one of the coefficients is almost zero, whereas the other one is of the order of $0.1 \Delta p$. With increasing irregularity both internal length parameters converge to a common value of about $\xi \approx 0.3 \Delta p$. Higher values of about one cell size ( $\Delta p$ in our case), as indicated by the experimental data of Hassanpour and Heppler (2017); Lakes (1991); McGregor and Wheel (2014), are not consistent with the couple stress patterns that derive from our microscale model. We will demonstrate this below.

For validation we use a direct comparison between averaged results from our microscale beam model and a finite element implementation of the Cosserat continuum that uses the material parameters of Fig. 4, averaged over all loading modes and system sizes.

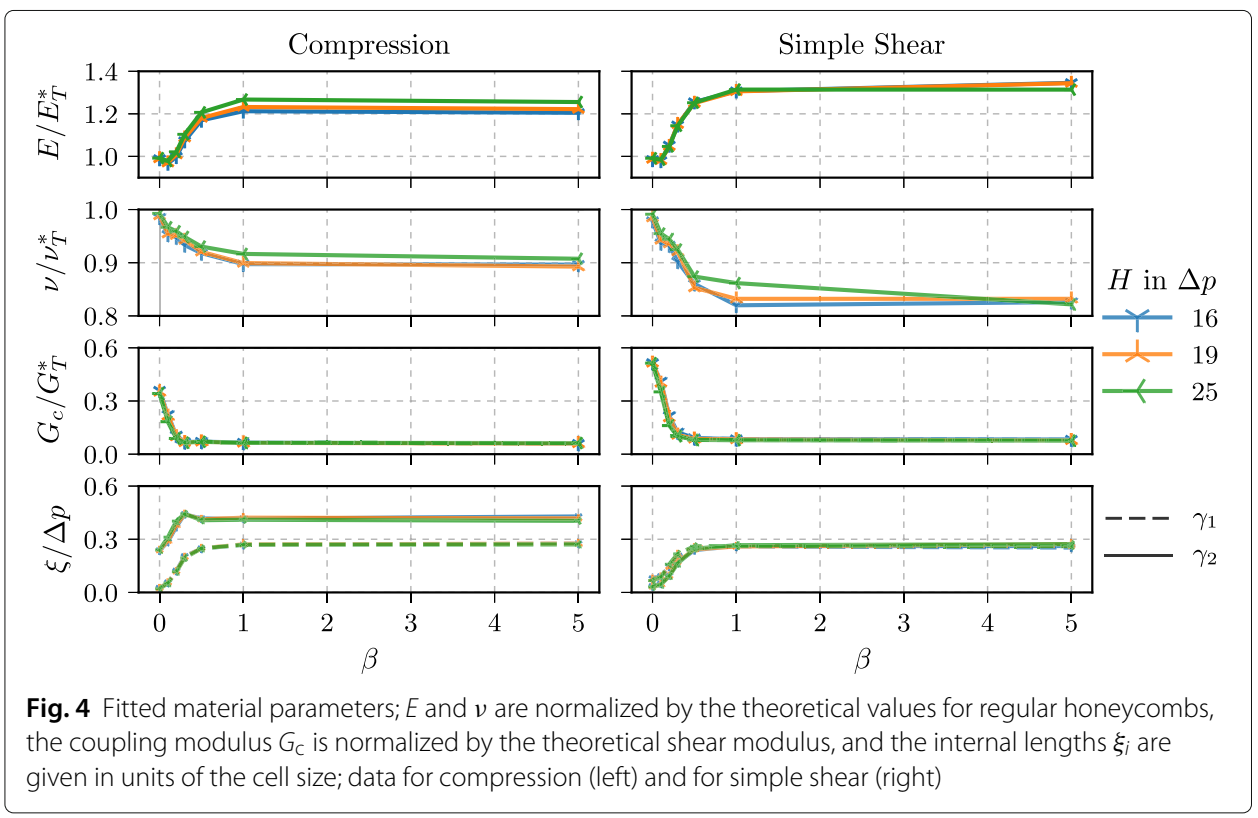


Macroscopic size effects show only if $\nabla \boldsymbol{u} \neq \nabla \boldsymbol{u}^{\top}$, which is not the case for uniaxial compression. (Note that it is nevertheless possible to determine internal length scale parameters by connecting the fluctuating local variables in the individual realizations which is what we have done above in case of compression). Therefore, only simple shear loading of the Cosserat medium is considered for validation purposes. We simulate a thin strip with the same width and height as the beam network and a thickness of $D=0.05 H$. By using only 1 element for the thickness, this loading state is similar to a plane stress loading. The intrinsic length requires a mesh which captures the thickness of the boundary layer. Thus the mesh is locally refined towards the surfaces such that the element width is significantly smaller than the internal length. The same boundary conditions as for the beam network are chosen (cf. Table 1) i.e. no in-plane Cosserat rotations and no $x$-displacements at the top and bottom and a $y$-displacement at the top which induces a engineering strain of $\varepsilon_{x y}^{*}=5 \%$. The $y$-displacement at the bottom boundary is set to zero whereas the out-of-plane rotations and displacements are unconstrained everywhere else.

As one can see from Fig. 5, left and centre, the stress distribution and the distribution of the rotations match very well. The maximum rotations are found along the free surfaces and gradually decrease towards the inner part of the specimen. The shear stresses show a characteristic distribution similar to the distribution for a classical continuum. From the free surfaces they gradually increase towards the center. The overall average of the Cosserat continuum system slightly under-predicts the average response. Couple stresses are concentrated in boundary layers at the top and bottom edges of the samples. Because the couple stresses $\mu$ are proportional to the rotation gradient $\nabla \omega$, the continuum and beam systems match badly near the specimen corners where both microrotations and micro-rotation gradients are high and rapidly alternating. In this region the continuous solutions of the Cosserat equations cannot easily match the continuized fields that by construction are piecewise constant over control volumes of finite size. In general, however, the agreement between the beam system and the Cosserat continuum is excellent. We quantify the overall agreement by evaluating the average moment of the couple stresses with respect to the symmetry axis of the system, $\langle y \boldsymbol{\mu}\rangle$ where $y=0$ marks the position of the horizontal symmetry axis (note that the direct average $\langle\boldsymbol{\mu}\rangle$ is zero for symmetry reasons and can therefore not be used for comparison purposes). Comparing $\langle y \boldsymbol{\mu}\rangle$ for the beam and Cosserat systems again demonstrates good quantitative agreement. For comparison we have also included computations which assume $\xi=\Delta p$ as suggested by Hassanpour and Heppler (2017); Lakes (1991); McGregor and Wheel (2014). The results of these computations are shown in Fig. 5, right. It is evident that they do not provide an adequate representation of the behavior of our beam model as the obtained values of $\langle y \boldsymbol{\mu}\rangle$ differ by one order of magnitude.

For further investigation we study row-wise averaged profiles of the rotations, the skewsymmetric stresses and the couple stresses:

$$
\begin{aligned}
\left\langle\omega_{z}\right\rangle_{\text {row }}(y) & =\frac{1}{W} \int_{0}^{W}\langle\omega\rangle_{\mathrm{E}}(x, y) \mathrm{d} x, \\
\left\langle S_{x y}\right\rangle_{\text {row }}(y) & =\frac{1}{W} \int_{0}^{W}\left\langle S_{x y}\right\rangle_{\mathrm{E}}(x, y) \mathrm{d} x, \\
\left\langle M_{z y}\right\rangle_{\text {row }}(y) & =\frac{1}{W} \int_{0}^{W}\left\langle M_{z y}\right\rangle_{\mathrm{E}}(x, y) \mathrm{d} x .
\end{aligned}
$$




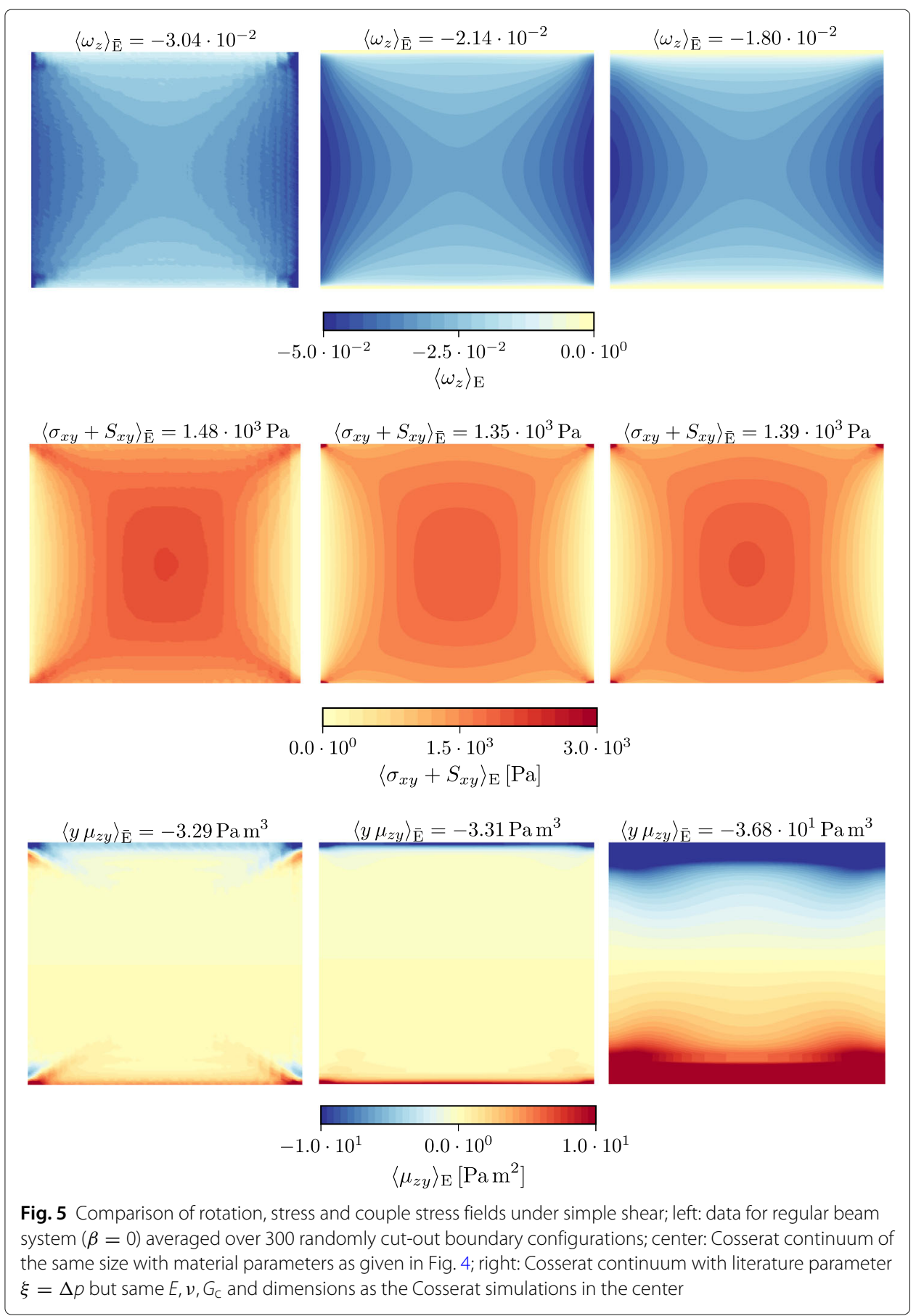

On the left of Fig. 6 one can see again the discussed effects. The rotation profiles match quite well, except for the outermost layer. The same is true for the couple stresses, which show very good agreement. The skew-symmetric stresses of the Cosserat continuum over predict the beam network response at the surface. It can be seen that the response of the beam network slightly oscillates near the boundaries which can be attributed to the spacing of the honeycombs and the fact that stresses and strains are assumed constant over the associated control volumes. 


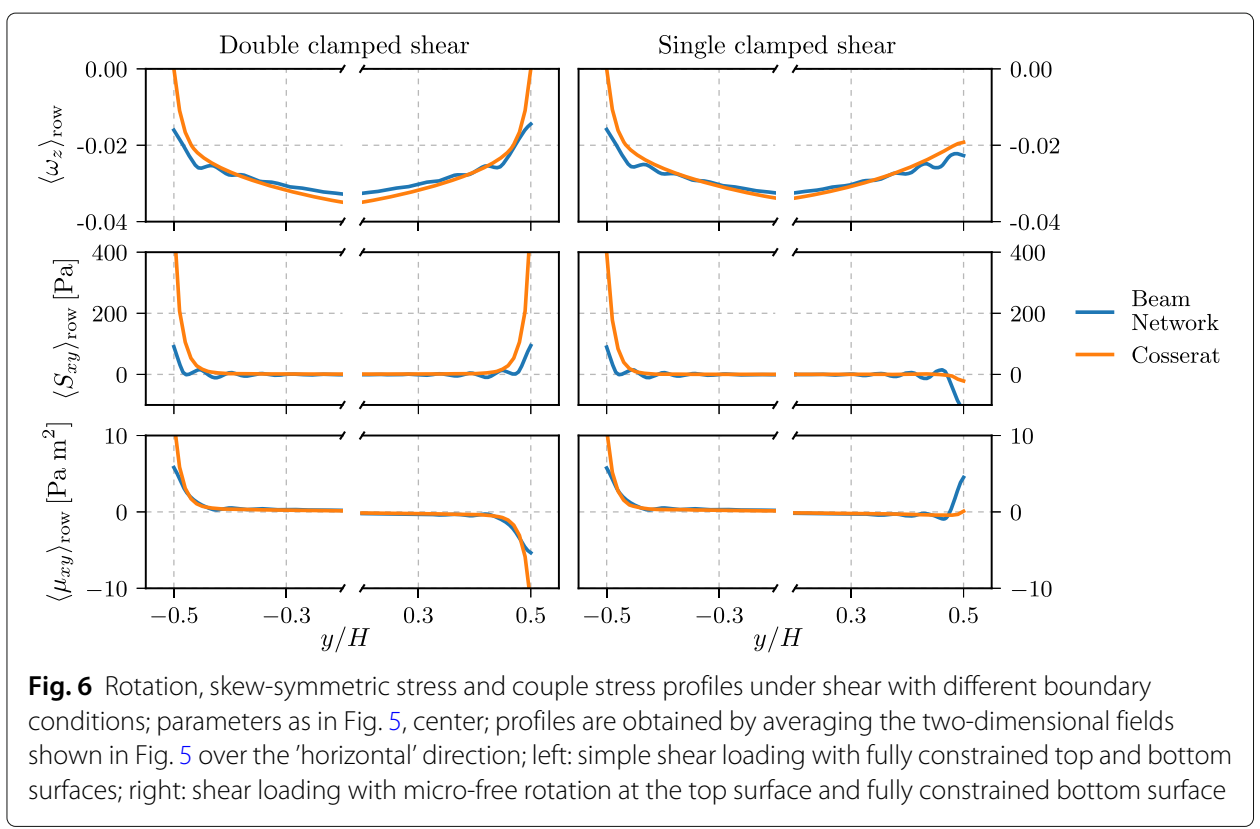

To test the predictive power of the parameterized Cosserat model we use the determined material parameters in a simulation with qualitatively different boundary conditions. Again a shear problem is considered, with the difference that the micro-rotations $\phi(H / 2)$ (beam system) and $\omega(H / 2)$ (Cosserat continuum) are now unconstrained at the top surface. In comparison to the doubly-clamped shear system this introduces an asymmetry into the problem which should reflect in the system response for the boundary layer as indeed observed in Fig. 6 on the right. The bottom surface $(y=-H / 2)$ shows an identical behavior as in the fully clamped system. In the Cosserat model the couple stress and skew-symmetric stress at the micro-free boundary $(y=H / 2)$ are zero up to numerical errors. The rotation at the top boundary is non-zero to fulfill the balance equations and higher order traction boundary condition $\boldsymbol{m}(y=H / 2)=0$. Whereas the rotations match quite well the couple stress and skew-symmetric stress differ Even though no additional fitting was done the Cosserat continuum matches well with the beam network, so that we conclude that the obtained parameter combination gives a reasonable approximation of the beam network and can be regarded as an informed way to determine Cosserat material properties.

Finally we compare the effective system response for the double-clamped, shear loaded systems. In analogy to the beam network (41) the effective system response of the continuum is

$$
C_{\mathrm{c}}^{*}=\frac{1}{A \varepsilon_{x y}^{*}} \int_{A} t_{x} \mathrm{~d} A .
$$

In Fig. 7 the averaged effective stiffness of all beam systems is for different irregularity parameter $\beta$ compared with result for the corresponding, fitted continuum models. The main trends, namely (i) the increase of overall stiffness with increasing degree of disorder and (ii) the existence of a size effect in the sense that systems of reduced height show a softer response, are well reproduced by the continuum model, though both the size effect and the disorder-induced stiffening are slightly more pronounced in the continuum model. 


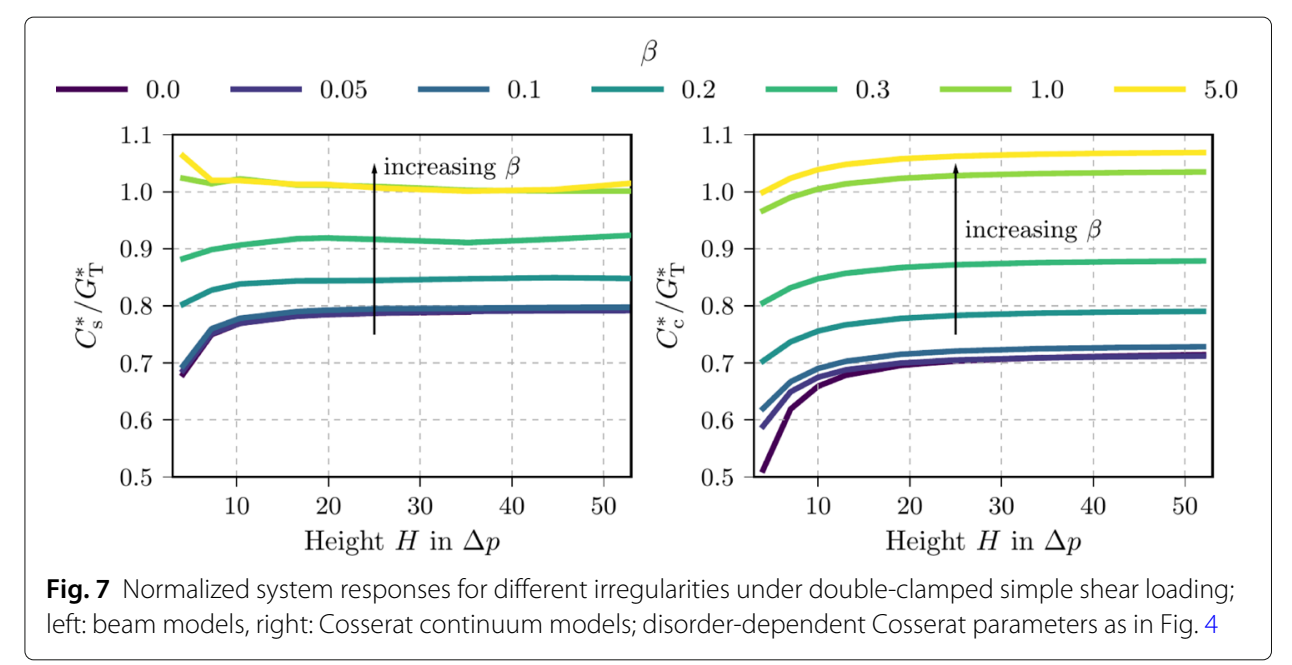

\section{Discussion and conclusions}

We presented a two-scale modeling approach for irregular cellular solids. On the microscale, we use beam models - an approach which implies a significant degree of idealization but is computationally much more efficient than for example modeling the microstructure with (Cauchy-) continuum elements. Computational cost is an important factor when dealing with strongly disordered microstructures where load is internally distributed in a strongly heterogeneous manner through force transmission chains (Liebenstein et al. 2017): for small sample sizes as considered here, such force transmission chains may span the entire sample and their stochastic character causes significant sample-to-sample variations. Hence, an efficient computational scheme is indispensable in order to capture the statistics of variations and to reliably determine the average behavior of samples with a given degree of irregularity. Despite the large sample-to-sample variability, the computational efficiency of beam models allows us to determine not only macroscopic stiffnesses but also smooth stress, strain, and rotation fields by averaging over sufficiently large ensembles of typically 6000 microstructure realizations for each system size and irregularity.

Our next step is to use the data from the micro-scale beam models in order to parametrize a macroscopic, Cosserat-type continuum model. The standard approach is a (bottom-up) homogenization where one seeks to derive macroscopic, effective materials properties through averaging the micro-heterogeneous material response over a representative volume element (RVE). We note that these methods may be problematic when it comes to assessing size dependent effects in structurally disordered materials which exhibit strongly heterogeneous stress and strain patterns with longrange correlations. This is the case in our micro-scale simulations: As demonstrated by Liebenstein et al. (2017), stress transmission in the strongly disordered beam models (high disorder parameter $\beta$ ) occurs through correlated stress transmission chains. In the regime of system sizes where size effects are relevant, in other words in the range of interest for a higher-order continuum model that incorporates internal length scales, these correlated stress transmission chains span the entire simulated sample. Since these chains are of stochastic nature, this leads to very significant sample-to-sample fluctuations in the 
macroscopic response. In other words, the simulated micro-sample sizes themselves are below the RVE scale.

In our case the transition from the beam model to the Cosserat continuum is on a local scale. First we map, in an energetically consistent manner that assumes the Cosserat continuum structure, the stress and deformation fields on the discrete beam level onto spatially continuous fields defined on control volumes that are characteristic of the particular microstructure. Determination of stresses and deformations is independent, and elastic constants are identified by seeking an optimum match between stress and conjugate strain variables through linear mappings on average over the ensemble of local control volumes. The approach used in the present study is contingent upon several conditions. (i) The material of the beam system must be isotropic. This is, for instance, not the case for an additively manufactured cellular structure where the deposition process imposes a preferential direction on the matrix material. (ii) The geometry of the individual control volumes (the cells) must be statistically isotropic. This feature is inherent in our construction of the beam network, which is based upon an isotropic honeycomb reference structure from which irregular structures are obtained via radial displacements of the cell seeds. (iii) The correlations between different control volumes must be statistically isotropic. In our construction of the beam network this is ensured by making the displacements of the cell seeds statistically independent and isotropic. (iv) There are no long-ranged geometrical correlations in the beam network. In fact, our construction method for the beam structure ensures that the shape fluctuations of different control volumes shapes are mutually uncorrelated beyond the nearest neighbors. This absence of correlations ensures that we can infer directly from the local behavior to the system scale behavior - which would be impossible if geometrical correlations between different control volumes were present.

Under these conditions, we can directly infer from the scale of the individual control volumes to the scale of the entire beam network. A remarkable consequence is that we can determine internal length scale parameters even in deformation settings where we do not expect macroscopic size effects, such as in uniaxial compression. The reason is that the local stress state is, owing to the micro-scale disorder of the material, always multi-axial such that rotation effects are present even in deformation settings that are macroscopically rotation free. Indeed the material constants we determine from the local fields in compression tests are in good agreement with those from simple shear tests. When used to evaluate size dependent response observed in different shear loadings, these parameters perform well even though they have not been fitted to reproduce shear data. The fact that one can use different uniform or non-uniform deformation settings to probe for the same parameters and then cross-validate them allows to ensure one is indeed dealing with material parameters and not parameters that characterize a specific deformation setting only.

Even though higher order continuum theories have their roots already 120 years ago they are still not commonly used in engineering applications. One reason is the difficulty to reliably determine constitutive parameters, of which in general theories there may be many. Once increasing computational power allows to efficiently simulate large systems and/or large ensembles of systems that are obviously inaccessible by experiment, this disadvantage may become less serious. In the presented work we showed, in a proofof-concept study, how Cosserat material parameters can be deduced from simulations of 
2D cellular structures. The parameters are obtained by a two step procedure. First the beam network is represented by an equivalent Cosserat continuum. From that the parameters are identified by a simple linear least square optimization approach, relying on local information only. The whole procedure works without fitting or adapting of parameters by hand. The beam modeling itself is purely linearly elastic, which of course means that the presented fitted parameters also assume a linearly elastic Cosserat continuum, i.e., we neither consider physically non-linear effects such as plasticity, beam breaking or beam contact, nor do we account for geometrically nonlinear phenomena such as beam buckling. However, we note that our continuization method can be used even if breaking of beams disrupts single control volumes and creates internal surfaces. Hence, it can be used to assess stress states in damaged or cracked samples and we plan to use the method to evaluate changes in stress transmission patterns occurring in damaged, brittle cellular structures in the approach to failure.

Of course there remain open questions. The present study has focused on statistically isotropic or near-isotropic systems with a single elementary length scale (the cell size). The method we propose has limitations when applied to anisotropic microstructures where there can be, besides anisotropy of the properties of the beam material two different sources of geometrical anisotropy: Anisotropy on the level of the individual microstructure element (e.g., an anisotropic average cell shape), and anisotropy in the arrangement of different elements, which might depend on additional correlation lengths (e.g., the shape of regions of higher-than-average or lower-than-average local density). The latter features cannot be captured by an approach which relies on information on the smallest microstrucural length scale, namely the individual cell or control volume, alone and may thus necessitate more sophisticated approaches. Further studies are also needed in order to investigate how the proposed method can be extended to three-dimensional open- or closed-cellular structures.

\section{Appendix}

\section{The statistical ensemble}

As discussed, a single realization of a beam system cannot be meaningfully compared with a continuum model, at least if the beam system is small. This poses problems if one wants to assess size effects, which of course become prominent only in small systems, and their modelling by generalized continuum models such as the Cosserat model. We therefore need to strictly distinguish between a system ensemble (which can be compared to a continuum model on any scale) and an individual system which cannot. In our simulations different beam system ensembles are characterized by different values of the common system size and degree of disorder (parameter $\beta$ ), other parameters (density, material parameters, beam parameters) being kept fixed. To obtain statistically reliable values for the response of the beam system ensemble, we average over multiple system realizations, a system realization consisting of a random cut-out from a random microstructure, with the size of the cut-out and the disorder parameter $\beta$ being kept fixed at the values characterizing the ensemble. We now focus on system-scale properties such as the effective shear stiffness in a shear test,

$$
C^{*}=\frac{1}{A \varepsilon_{x y}^{*}} \sum_{k=1}^{N_{\mathrm{R}}} F_{x}^{k},
$$


where

$$
\varepsilon_{x y}^{*}=\frac{\left|u_{x}(y=H / 2)-u_{x}(y=-H / 2)\right|}{H}
$$

is the effective engineering strain and $F_{x}$ are the load induced reaction forces in $x$ direction of all the beams $N_{\mathrm{R}}$ at the loaded top and bottom surfaces. All system realizations are subjected to the same boundary loading. The resulting response values will scatter. We denote by $\left\langle C^{*}\right\rangle_{\mathrm{S}}$ the average over $N_{\mathrm{CO}}$ different cut-outs from the same microstructure, and by $s_{\mathrm{CO}}$ the corresponding standard deviation. Furthermore, we denote by $\left\langle C^{*}\right\rangle$ the average of $\left\langle C^{*}\right\rangle_{\mathrm{S}}$ over $N_{\mathrm{S}}$ different microstructure realizations and by $s_{\mathrm{S}}$ the corresponding standard deviation. We observe that the statistical deviations from the respective mean are independent between different cut-outs, and between different realizations. However, strength variations in a given cut-out may be realization dependent, indicating correlation. An upper estimate for the total scatter is then given by

$$
s=s_{\mathrm{S}} / \sqrt{N_{\mathrm{S}}}+s_{\mathrm{CO}} / \sqrt{N_{\mathrm{CO}}}
$$

We adjust the numbers of realizations, and of cut-outs, in such a manner that this quantity remains consistently below $1 \%$ of the mean,

$$
s \leq 0.01\left\langle C^{*}\right\rangle .
$$

For highly ordered systems, where $s_{\mathrm{S}}$ is small or even zero (regular honeycombs) but $s_{\mathrm{CO}}$ can be significant for small systems, this implies a high value of $N_{\mathrm{CO}}$, typically $N_{\mathrm{CO}} \approx$ 400. For small and strongly disordered systems, conversely, one uses a high value of $N_{\mathrm{S}}$ (typically 400 ) but a small value of $N_{\mathrm{CO}}$, typically $N_{\mathrm{CO}} \approx 20$.

\section{Acknowledgements}

The authors acknowledge funding by DFG under Grant no. 1 Za 171-9/1. M.Z. also acknowledges support by the Chinese government under the Program for the Introduction of Renowned Overseas Professors (MS2016XNJT044).

\section{Authors' contributions}

$S L$ devised the continuization scheme, set up and performed all simulations and statistical analysis, and drafted the first version of the manuscript. MZ assisted in the final formulation of the continuization scheme and suggested the fitting scheme for determination of elastic parameters. Both authors jointly wrote the final manuscript. Both authors read and approved the final manuscript.

\section{Competing interests}

The authors declare that they have no competing interests.

\section{Publisher's Note}

Springer Nature remains neutral with regard to jurisdictional claims in published maps and institutional affiliations.

\section{Author details}

${ }^{1}$ Institute of Materials Simulation (WW8), Friedrich-Alexander University Erlangen-Nürnberg (FAU), Dr.-Mack-Strasse 77, 90762 Fürth, Germany. ${ }^{2}$ Department of Engineering and Mechanics, Southwest Jiaotong University, Chengdu, People's Republic of China.

Received: 15 August 2017 Accepted: 19 December 2017

Published online: 31 January 2018

References

H Altenbach, S Forest, A Krivtsov, Generalized Continua as Models for Materials, 1st edn. (Springer, Berlin, Heidelberg, 2013)

W Anderson, R Lakes, Size effects due to cosserat elasticity and surface damage in closed-cell polymethacrylimide foam. J. Mater. Sci. 29(24), 6413-6419 (1994)

E Andrews, G Gioux, P Onck, L Gibson, Size effects in ductile cellular solids. part ii: experimental results. Int. J. Mech. Sci. 43(3), 701-713 (2001)

Z Bažant, M Christensen, Analogy between micropolar continuum and grid frameworks under initial stress. Int. J. Solids Struct. 8(3), 327-346 (1972)

A Beveridge, M Wheel, D Nash, The micropolar elastic behaviour of model macroscopically heterogeneous materials. Int. J. Solids Struct. 50(1), 246-255 (2013)

B Boots, The arrangement of cells in "random" networks. Metallography. 15(1), 53-62 (1982) 
C Chen, R Lakes, Holographic study of conventional and negative poisson's ratio metallic foams: elasticity, yield and micro-deformation. J. Mater. Sci. 26(20), 5397-5402 (1991)

C Chen, T Lu, N Fleck, Effect of imperfections on the yielding of two-dimensional foams. J. Mech. Phys. Solids. 47(11), 2235-2272 (1999)

E Cosserat, F Cosserat, Théorie des corps déformables. Hermann et fils, Paris. 3, 17-29 (1909)

G Cowper, The shear coefficient in timoshenko's beam theory. J. Appl. Mech. 33(2), 335-340 (1966)

$S$ Diebels, A Geringer, Micromechanical and macromechanical modelling of foams: Identification of cosserat parameters. ZAMM - J. Appl. Math. Mech. Z. für Angew. Math. Mech. 94(5), 414-420 (2014)

$\mathrm{S}$ Diebels, H Steeb, The size effect in foams and its theoretical and numerical investigation. Proc. Roy. Soc. Lond. A Math. Phys. Eng. Sci. 458(2028), 2869-2883 (2002)

F Dos Reis, J Ganghoffer, Construction of micropolar continua from the asymptotic homogenization of beam lattices. Comput. Struct. 112, 354-363 (2012)

AC Eringen, Mechanics of micromorphic materials. (Springer, Berlin Heidelberg, Berlin, Heidelberg, 1966), pp. 131-138

AC Eringen, Microcontinuum Field Theories - Foundations and Solids. (Springer, New York, 1999)

AC Eringen, E Suhubi, Nonlinear theory of simple micro-elastic solids-i. Int. J. Eng. Sci. 2(2), 189-203 (1964)

F Feyel, A multilevel finite element method (fe2) to describe the response of highly non-linear structures using generalized continua.Comput. Methods Appl. Mech. Eng. 192(28), 3233-3244 (2003). Multiscale Computational Mechanics for Materials and Structures

S Forest, Mechanics of generalized continua: construction by homogenizaton. J. Phys. IV. 8(PR4), Pr4-39 (1998)

S Forest, Micromorphic approach for gradient elasticity, viscoplasticity, and damage. J. Eng. Mech. 135(3), 117-131 (2009)

S Forest, K Sab, Cosserat overall modeling of heterogeneous materials. Mech. Res. Commun. 25(4), 449-454 (1998)

S Forest, F Pradel, K Sab, Asymptotic analysis of heterogeneous cosserat media. Int. J. Solids Struct. 38(26), 4585-4608 (2001)

R Gauthier, W Jahsman, A quest for micropolar elastic constants. J. Appl. Mech. 42(2), 369-374 (1975)

$\mathrm{S}$ Ghosh, K Lee, S Moorthy, Two scale analysis of heterogeneous elastic-plastic materials with asymptotic homogenization and voronoi cell finite element model. Comput. Methods Appl. Mech. Eng. 132(1), 63-116 (1996)

L Gibson, M Ashby, Cellular Solids: Structure and Properties. Cambridge Solid State Science Series, (Cambridge University Press, Cambridge, 1999)

S Hassanpour, GR Heppler, Micropolar elasticity theory: a survey of linear isotropic equations, representative notations, and experimental investigations. Math. Mech. Solids. 22(2), 224-242 (2017)

R Jänicke, S Diebels, HG Sehlhorst, A Düster, Two-scale modelling of micromorphic continua. Contin. Mech. Thermodyn. 21(4), 297-315 (2009)

N Kirchner, P Steinmann, Mechanics of extended continua: modeling and simulation of elastic microstretch materials. Comput. Mech. 40(4), 651-666 (2007)

$\checkmark$ Kouznetsova, M Geers, W Brekelmans, Multi-scale second-order computational homogenization of multi-phase materials: a nested finite element solution strategy. Comput. Methods Appl. Mech. Eng. 193(48), 5525-5550 (2004)

RS Kumar, DL McDowell, Generalized continuum modeling of 2-d periodic cellular solids. Int. J. Solids Struct. 41 (26), 7399-7422 (2004)

R Lakes, Size effects and micromechanics of a porous solid. J. Mater. Sci. 18(9), 2572-2580 (1983)

R Lakes, Experimental microelasticity of two porous solids. Int. J. Solids Struct. 22(1), 55-63 (1986)

$\mathrm{R}$ Lakes, Experimental micro mechanics methods for conventional and negative poisson's ratio cellular solids as cosserat continua. ASME Trans. J. Eng. Mater. Technol. 113, 148-155 (1991)

S Liebenstein, S Sandfeld, M Zaiser, Modelling elasticity of open cellular foams: Size effects and disorder (2017). submitted to International Journal of Solids and Structures preprint: https://arxiv.org/abs/1604.03589. Accessed 07 Jan 2018

M McGregor, MA Wheel, On the coupling number and characteristic length of micropolar media of differing topology. Proc. Roy. Soc. Lond. A Math. Phys. Eng. Sci. 470(20140150) (2014)

RJ Mora, AM Waas, Evaluation of the micropolar elasticity constants for honeycombs. Acta Mech. 192(1), 1 (2007)

KE Nady, FD Reis, I Ganghoffer, Computation of the homogenized nonlinear elastic response of $2 d$ and $3 d$ auxetic structures based on micropolar continuum models. Compos. Struct. 170, 271-290 (2017)

PR Onck, Cosserat modeling of cellular solids. Compt. Rendus Mecanique. 330(11), 717-722 (2002)

M Ostoja-Starzewski, Lattice models in micromechanics. Appl. Mech. Rev. 55(1), 35-60 (2002)

M Ostoja-Starzewski, Microstructural randomness and scaling in mechanics of materials. (CRC Press, Boca Raton, 2007)

MJ Silva, WC Hayes, LJ Gibson, The effects of non-periodic microstructure on the elastic properties of two-dimensional cellular solids. Int. J. Mech. Sci. 37(11), 1161-1177 (1995)

C Tekoğlu, P Onck, Size effects in the mechanical behavior of cellular materials. J. Mater. Sci. 40(22), 5911-5917 (2005)

C Tekoğlu, PR Onck, Size effects in two-dimensional voronoi foams: A comparison between generalized continua and discrete models. J. Mech. Phys. Solids. 56(12), 3541-3564 (2008)

C Tekoğlu, L Gibson, T Pardoen, P Onck, Size effects in foams: Experiments and modeling. Prog. Mater. Sci. 56(2), 109-138 (2011)

P Trovalusci, A Pau, Derivation of microstructured continua from lattice systems via principle of virtual works: the case of masonry-like materials as micropolar, second gradient and classical continua. Acta Mech. 225(1), 157-177 (2014)

P Trovalusci, ML De Bellis, R Masiani, A multiscale description of particle composites: From lattice microstructures to micropolar continua. Compos. B Eng. 128, 164-173 (2017)

MWD Van Der Burg, V Shulmeister, E Van Der Geissen, R Marissen, On the linear elastic properties of regular and random open-cell foam models. J. Cell. Plast. 33(1), 31-54 (1997)

W Warren, A Kraynik, Foam mechanics: the linear elastic response of two-dimensional spatially periodic cellular materials. Mech. Mater. 6(1), 27-37 (1987)

M Wheel, J Frame, P Riches, Is smaller always stiffer? On size effects in supposedly generalised continua. Int. J. Solids Struct. 67-68, 84-92 (2015)

C Woźniak, Surface lattice structures (in polish). (Polish Scientific Publishers (PWN), Warsaw, 1970)

$J$ Yang, RS Lakes, Experimental study of micropolar and couple stress elasticity in compact bone in bending. J. Biomech. 15(2), 91-98 (1982) 
H Zhu, J Hobdell, A Windle, Effects of cell irregularity on the elastic properties of open-cell foams. Acta Mater. 48(20), 4893-4900 (2000)

H Zhu, S Thorpe, A Windle, The geometrical properties of irregular two-dimensional voronoi tessellations. Philos. Mag. A. 81(12), 2765-2783 (2001)

H Zhu, S Thorpe, A Windle, The effect of cell irregularity on the high strain compression of $2 d$ voronoi honeycombs. Int. J. Solids Struct. 43(5), 1061-1078 (2006)

Submit your manuscript to a SpringerOpen ${ }^{\circ}$ journal and benefit from:

- Convenient online submission

- Rigorous peer review

- Open access: articles freely available online

- High visibility within the field

- Retaining the copyright to your article

Submit your next manuscript at $\gg$ springeropen.com 